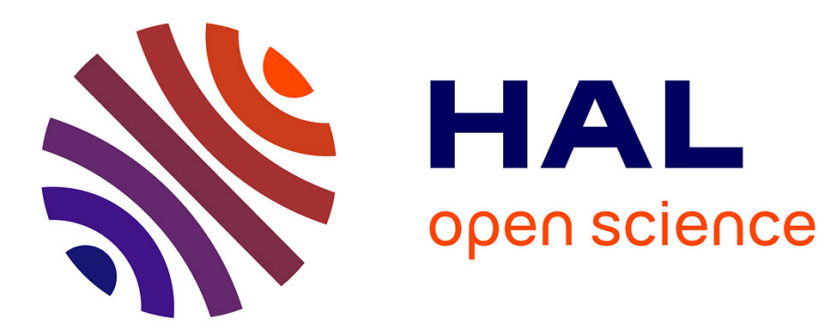

\title{
Phosphate hydrogénophosphate hydrate de manganèse
} Y Gerault, A Riou, Yannick Cudennec

\section{To cite this version:}

Y Gerault, A Riou, Yannick Cudennec. Phosphate hydrogénophosphate hydrate de manganèse. Acta Crystallographica Section C : Crystal Structure Communications [1968-2013], 1987, 43, pp.1829 - 1830. $10.1107 / \mathrm{s} 0108270187090000$. hal-03226039

\section{HAL Id: hal-03226039 \\ https://hal.science/hal-03226039}

Submitted on 13 May 2021

HAL is a multi-disciplinary open access archive for the deposit and dissemination of scientific research documents, whether they are published or not. The documents may come from teaching and research institutions in France or abroad, or from public or private research centers.
L'archive ouverte pluridisciplinaire HAL, est destinée au dépôt et à la diffusion de documents scientifiques de niveau recherche, publiés ou non, émanant des établissements d'enseignement et de recherche français ou étrangers, des laboratoires publics ou privés. 
Acta Cryst. (1987). C43, 1829-1830.

https://doi.org/10.1107/S0108270187090000

\title{
Phosphate Hydrogénophosphate Hydrate de Manganèse
}

\author{
Par Y. Gérault, A. Riou et Y. Cudennec \\ Laboratoire de Chimie des Matériaux Inorganiques et de Cristallographie, 20 avenue des Buttes de Coësmes, \\ 35043 Rennes CEDEX, France.
}

(Reçu le 8 décembre 1986, accepté le 10 avril 1987)

\begin{abstract}
Mn}_{5}\left(\mathrm{HPO}_{4}\right)_{2}\left(\mathrm{PO}_{4}\right)_{2} .4 \mathrm{H}_{2} \mathrm{O}, \mathrm{M}_{\mathrm{r}}=728.6$, monoclinic, $C 2 / c, a=17.587$ (4), $b=9.127$ (3), $c$ $=9.497$ (5) $A, \beta=96.68(3)^{\circ}, V=1514$ (1) $\AA^{3}, D_{m}=3.19(1), D_{x}=3.19 \mathrm{Mg} \cdot \mathrm{m}^{-3}, Z=4, \mathrm{Mo} \mathrm{K \alpha}, \lambda$ $=0.71069 \AA, \mu=4.47 \mathrm{~mm}^{-1}, F(000)=1420, T=130 \mathrm{~K}, R=0.023$ for 1581 reflections. The structure is isotypic with hureaulite. Apart from alkaline-earth metals which are often found in weak concentrations in natural minerals, it appears that compounds of general formula:
\end{abstract}

$\left(\mathrm{Mn}_{1-\mathrm{x}} \mathrm{Fe}_{\mathrm{x}}\right)_{5}\left(\mathrm{HPO}_{4}\right)_{2}\left(\mathrm{PO}_{4}\right)_{2} .4 \mathrm{H}_{2} \mathrm{O}$ probably exist for all $\mathrm{x}$ in the range $0-1$.

Partie expérimentale. Une étude récente de phosphates de métaux divalents (Cudennec, Riou \& Gerault, 1986) a permis, entre autre, la préparation du composé $\mathrm{Mn}_{5}\left(\mathrm{HPO}_{4}\right)_{2}\left(\mathrm{PO}_{4}\right)_{2} \cdot 4 \mathrm{H}_{2} \mathrm{O}$. Les monocristaux obtenus se présentent sous la forme d'aiguilles pratiquement incolores. Les paramètres cristallins ont été déterminés et affinés sur un diffractomètre automatique à partir de 25 réflexions et la masse volumique mesurée par la méthode de la poussée d'Archimède. La similitude des résultats obtenus avec ceux observés dans l'étude du minéral naturel la hureaulite nous a conduit à envisager l'hypothèse d'une isotypie structurale. Cette famille aurait donc comme formulation générale $\left(\mathrm{Mn}_{1-\mathrm{x}} \mathrm{Fe}_{\mathrm{x}}\right)_{5}\left(\mathrm{HPO}_{4}\right)_{2}\left(\mathrm{PO}_{4}\right)_{2} \cdot 4 \mathrm{H}_{2} \mathrm{O}$. La détermination structurale sur monocristal de tels composés a été réalisée pour $\mathrm{x}=0.26$ (Moore \& Araki, 1973) et $\mathrm{x}=0.11$ (Menchetti \& Sabelli, 1973). Il nous a semblé intéressant de déterminer la structure de $\mathrm{Mn}_{5}\left(\mathrm{HPO}_{4}\right)_{2}\left(\mathrm{PO}_{4}\right)_{2} .4 \mathrm{H}_{2} \mathrm{O}$ qui pourrait alors être considérée comme une hureaulite de manganèse pur.

Les 1866 intensités indépendantes $\left(2 \theta<56^{\circ}\right.$; h 0 --- 23, k 0 --- 12, $1-11$--- 11) utilisées dans cette détermination structurale ont été collectées sur un diffractomètre automatique CAD-4 EnrafNonius. La stabilité dans le temps a été contrôlée périodiquement par le suivi de l'évolution de trois réflexions standards, la variation d'intensité pendant les 23 heures d'enregistrement est de $0.5 \%$. Afin de diminuer les valeurs des coefficients d'agitation thermique, et, ainsi mieux positionner les hydrogènes, l'enregistrement a été effectué à basse température $(130 \mathrm{~K})$. Les intensités brutes ont été corrigées des facteurs de Lorentz et de polarisation, par contre, aucune correction d'absorption n'a été nécessaire compte tenu des dimensions du cristal $(0.650 \times 0.050 \times 0.075 \mathrm{~mm})$. La détermination structurale a été faite sur la base de l'isotypie avec la hureaulite naturelle. Les calculs ont été effectués sur un ordinateur PDP 11/60 avec la chaîne de programme Enraf-Nonius SDP (Frenz, 1978). Pour l'affinement final des 148 variables, basé sur une méthode de moindres carrés à 
matrice totale, 1581 observations ont été retenues comme significatives. Les valeurs des coefficients de confiance se stabilisent à $R(F)=0,023$ et $w R=0,036\left(p=0,06, \Delta / \sigma<0,37, \Delta p<0,387\right.$.e $\left.\AA^{-3}\right)$. Les valeurs finales des coordonnées atomiques et des coefficients d'agitation thermique sont consignées au Tableau 1.*

* Les listes des facteurs de structure et des facteurs d'agitation thermique anisotrope ont été déposées au dépôt d'archives de la British Library Document Supply Centre (Supplementary Publication No. SUP 43957: 10 pp.). On peut en obtenir des copies en s'adressant à: The Executive Secretary, International Union of Crystallography, 5 Abbey Square, Chester CH1 2HU, Angleterre.

Littérature associée. Les faibles écarts observés entre nos résultats et ceux de Moore \& Araki (1973) et Menchetti \& Sabelli (1973) confirment le bien fondé de notre hypothèse d'isotypie. Le domaine d'existence des minéraux naturels se situerait pour des valeurs de x comprises entre 0,09 et 0,26 (Menchetti \& Sabelli, 1973). Un récent travail (Corbin, Whitney, Fultz, Stucky, Eddy \& Cheetham, 1986) a permis la mise en évidence par voie de synthèse d'un composé du même type pour lequel $\mathrm{x}=1$.

\section{Références}

Corbin, D. R., Whitney, J. F., Fultz, W. C., Stucky, G. D., EdDy, M. M. \& Cheetham, A. K. (1986). Inorg. Chem. 25, 2279-2280.

Cudennec, Y., Riou, A. \& Gerault, Y. (1986). C.R. Acad. Sci. Ser. II, 302, 1149-1154.

Frenz, B. A. (1978). Computing in Crystallography, édite par H. SCHENK, R. Olthof-HAZEKAMP, H. van Koningsveld \& G. C. BAssi, pp. 64-71. Delft Univ. Press.

Menchetti, S. \& Sabelli, C. (1973). Acta Cryst. B29, 25412548.

Moore, P. B. \& Araki, T. (1973). Am. Mineral. 58, 302-307. 
Tableau 1. Paramètres atomiques et coefficients d'agitation thermique isotrope équivalents de $\mathrm{Mn}_{5}\left(\mathrm{HPO}_{4}\right)_{2}\left(\mathrm{PO}_{4}\right)_{2} \cdot 4 \mathrm{H}_{2} \mathrm{O}$

$$
B_{\text {éq }}=\frac{4}{3} \sum_{i} \sum_{j} \beta_{i j} \mathbf{a}_{i} \cdot \mathbf{a}_{j} \text {. }
$$

\begin{tabular}{lllll} 
& \multicolumn{1}{c}{$x$} & $y$ & \multicolumn{1}{c}{$z$} & $B_{\text {ég }}\left(\AA^{2}\right)$ \\
$\mathrm{Mn}(1)$ & 0,000 & $0,89716(5)$ & 0,250 & $0,404(8)$ \\
$\mathrm{Mn}(2)$ & $0,18207(2)$ & $0,41117(4)$ & $0,31526(4)$ & $0,452(6)$ \\
$\mathrm{Mn}(3)$ & $0,17439(2)$ & $0,02784(4)$ & $0,36531(4)$ & $0,440(6)$ \\
$\mathrm{P}(1)$ & $0,08274(3)$ & $0,17952(6)$ & $0,08980(6)$ & $0,338(9)$ \\
$\mathrm{P}(2)$ & $0,33943(3)$ & $0,23913(6)$ & $0,37197(6)$ & $0,311(9)$ \\
$\mathrm{O}(1)$ & $0,0118(1)$ & $0,2851(2)$ & $0,0808(2)$ & $0,67(3)$ \\
$\mathrm{O}(2)$ & $0,07703(9)$ & $0,0674(2)$ & $0,2068(2)$ & $0,58(3)$ \\
$\mathrm{O}(3)$ & $0,1547(1)$ & $0,2714(2)$ & $0,1326(2)$ & $0,59(3)$ \\
$\mathrm{O}(4)$ & $0,4161(1)$ & $0,3919(2)$ & $0,0555(2)$ & $0,57(3)$ \\
$\mathrm{O}(5)$ & $0,1638(1)$ & $0,2366(2)$ & $0,4673(2)$ & $0,50(3)$ \\
$\mathrm{O}(6)$ & $0,2982(1)$ & $0,0959(2)$ & $0,3265(2)$ & $0,53(3)$ \\
$\mathrm{O}(7)$ & $0,29857(9)$ & $0,3666(2)$ & $0,2888(2)$ & $0,58(3)$ \\
$\mathrm{O}(8)$ & $0,4244(1)$ & $0,2332(2)$ & $0,3425(2)$ & $0,48(3)$ \\
$\mathrm{O}(9)$ & $0,2590(1)$ & $0,0806(2)$ & $0,0304(2)$ & $0,64(3)$ \\
$\mathrm{O}(10)$ & $0,4228(1)$ & $0,0122(2)$ & $0,1507(2)$ & $0,95(3)$ \\
$\mathrm{H}(1)$ & $0,481(3)$ & $0,224(5)$ & $0,505(5)$ & $*$ \\
$\mathrm{H}(2)$ & $0,743(3)$ & $0,349(5)$ & $0,578(5)$ & $*$ \\
$\mathrm{H}(3)$ & $0,712(3)$ & $0,123(5)$ & $0,508(5)$ & $*$ \\
$\mathrm{H}(4)$ & $0,560(3)$ & $0,089(5)$ & $0,279(5)$ & $*$ \\
$\mathrm{H}(5)$ & $0,461(3)$ & $0,032(5)$ & $0,631(5)$ & $*$
\end{tabular}

* Atomes affinés isotropiquement: $B=4,0 \AA^{2}$. 